\title{
СПОСОБ МАТРИЧНОГО РАСЧЕТА СЛОЖНЫХ ХИМИКО-ТЕХНОЛОГИЧЕСКИХ СИСТЕМ
}

\author{
(Представил О. Эйзен)
}

При оптимизации сложных химико-технологических систем (СХTC) один из способов расчета основан на решении уравнений математических моделей всех элементов СХТС и учете связей переменных в разных моделях. Такой подход гарантирует максимальную точность, но обычно возникает огромная система уравнений, решение которой требует значительных расчетных мощностей и времени. Методы и алго. ритмы для такого подхода разработаны для больших ӘВМ ['].

Другим методом расчета является матричный. В этом случае математическая модель СХТС (эквивалентная матрица преобразования системы) получается объединением матриц преобразования (МП) отдельных технологических операторов (ТО) в соответствии со структурной схемой СХТС. Такой способ расчета СХТС позволяет получить решение безытерационным путем и сочетает в себе удовлетворительную точность и возможность полной формализации расчетных процедур. Необходимо отметить, что матричный метод расчета СХТС предусматривает наличие линейных или слабонелинейных характеристик ТО. В противном случае нужно специально оценивать область линеаризации и проводить итерационные процедуры, корректируя элементы МП TO.

Матричный метод расчета СХТС, предложенный в [2], требует предварительного преобразования структурной схемы, которое затруднительно запрограммировать для ЭВМ.

Нами предлагается способ, который хорошо поддается программированию и позволяет рассчитывать СХТС полностью формализованно. Для снижения размерности матриц все переменные разделены на два типа: находящиеся в «межблоковых потоках»и находящиеся только в пределах одного блока. МП можно составить так, чтобы они учитывали переменные только первого типа. Для переменных второго типа можно составить свои МП и т. н. дополнительные потоки. Для экономии машинного времени расчет СХTC проводится в два этапа. Сначала составляются формулы, которые зависят только от структуры схемы. Они закладываются в память ЭВМ. А затем, при расчете конкретной ситуации, в эти готовые формулы подставляются соответствующие матричные элементы и проводится расчет. Такой способ особенно удобен в том случае, когда расчет СХТС нужно проводить многократно.

Опишем наш способ расчета. Пусть для каждого блока СХТС известна матрица преобразования $M$ :

$$
y=M x,
$$

где $x-$ входной поток в блок, $y-$ выходной поток из блока. 
Эквивалентная матрица преобразования (ЭМП) системы $C$ выражает зависимость всех переменных СХТС. Эту зависимость можно записать в виде

$$
x_{\mathrm{Bdx}}=C \times\left(x_{\mathrm{Bx}}, y_{1}, \ldots, y_{L}\right),
$$

где $x_{\text {вых }}$ - выходной поток из системы, $x_{\text {вх }}$ - основной входной поток в систему, $y_{1}, \ldots, y_{L}-$ входные потоки.

Нами использованы результаты структурного анализа схемы СХТС, где выделены и упорядочены комплексы и внутри каждого комплекса определены оптимальные совокупности потоков, разрыв которых превращает комплекс в разомкнутую схему. В качестве критерия оптимальности в данном случае разумно выбрать число разрываемых потоков.

ЭМП можно найти для каждого комплекса отдельно. Выходным потоком комплекса является поток, соединяющий рассматриваемый комплекс со следующим за ним комплексом. Так как выходных потоков из комплекса может быть несколько, то у комплекса может быть и несколько ЭМП. Общая ЭМП СХТС получается умножением найденных матриц в соответствующем порядке. Таким образом, в дальнейшем можно рассматривать нахождение ЭМП одного комплекса.

Формулы сохраняются в ӘВМ в виде последовательности цифр. Для каждого потока и матрицы системы имеется однозначный символ некоторая цифра. Свои символы имеют и матричные операции - сложение, вычитание, умножение и обращение. В формулах символ операции стоит после символов матриц и векторов. Таким же образом надо представлять и вводимые формулы (формулы неосновных входных потоков).

Приведем алгоритм нашего способа. На первом шаге надо составить систему линейных уравнений, которая выражает взаимосвязи между потоками комплекса. Уравнения этой системы должны содержать разрываемые потоки, основные входные потоки (их может быть несколько), входные потоки (они представлены формулами, в которые входят другие потоки), выходные потоки (их может быть тоже несколько) и дополнительные (напр., потоки, которые содержатся в формулах потоков).

Система линейных уравнений такова:

$$
\begin{aligned}
& x_{i_{1}}=M_{1,1} x_{i_{1}}+\ldots+M_{1, k} x_{i_{k}}+M_{1, k+1} x_{\mathrm{Bx}_{1}}+\ldots+M_{1, k+p} x_{\mathrm{Bx}_{p}}+ \\
& +M_{1, k+p+1} y_{1}+\ldots+M_{1, k+p+t} y_{t}, \\
& x_{i k}=M_{k, 1} x_{i_{1}}+\ldots+M_{k, k} x_{i_{k}}+M_{k, k+1} x_{\mathrm{Bx}_{1}}+\ldots+M_{k, k+p} x_{\mathrm{Bx}_{p}}+ \\
& +M_{k, k+p+1} y_{1}+\ldots+M_{k, k+p+t} y_{t} \text {, } \\
& x_{\mathrm{Bdx}}=M_{k+1,1} x_{i_{1}}+\ldots+M_{k+1, k} x_{i_{k}}+M_{k+1, k+1} x_{\mathrm{Bx}_{1}}+\ldots+M_{k+1, k+p} x_{\mathrm{Bx}_{p}}+ \\
& +M_{k+1, k+p+1} y_{1}+\ldots+M_{k+1, k+p+t} y_{t} \text {, }
\end{aligned}
$$

$x_{\mathrm{Bdx}}=M_{k+m, 1} x_{i_{1}}+\ldots+M_{k+m, k} x_{i_{k}}+M_{k+m, k+1} x_{\mathrm{Bx}_{1}}+\ldots+M_{k+m, k+p} x_{\mathrm{Bx}_{p}}+$

$$
+M_{k+m, k+p+1} y_{1}+\ldots+M_{k+m, k+p+t} y_{t},
$$

$x_{j_{1}}=M_{k+m+1,1} x_{i_{1}}+\ldots+M_{k+m+1, k} x_{i_{k}}+M_{k+m+1, k+1} x_{\mathrm{Bx}_{1}}+\ldots+M_{k+m+1, k+p} x_{\mathrm{Bx}_{p}}+$ $+M_{k+m+1, k+p+1} y_{1}+\ldots+M_{k+m+1, k+p+t} y_{t}$,

$x_{j q}=M_{k+m+q, 1} x_{i_{1}}+\ldots+M_{k+m+q, k} x_{i_{k}}+M_{k+m+q, k+1} x_{\mathrm{BX}_{\mathrm{t}}}+\ldots+M_{k+m+q, k+p} x_{\mathrm{Bx}_{p}}+$ $+M_{k+m+q, k+p+1} y_{1}+\ldots+M_{k+m+q, k+p+t} y_{t}$, 
где $x_{i_{1}}, \ldots, x_{i_{k}}-$ разрываемые потоки, $x_{\mathrm{Bx}_{1}}, \ldots, x_{\mathrm{Bx}_{p}}-$ основные входные потоки, $y_{1}, \ldots, y_{t}-$ входные потоки, $x_{\text {вых }}, \ldots, x_{\text {вых }}-$ выходные потоки, $x_{j_{1}}, \ldots, x_{j q}$ - дополнительные потоки.

Формулы коэффициентов $M_{i, j}(i=1, \ldots, k+m+q, j=1, \ldots, k+p+t)$ находят в соответствии со структурой схемы между потоками $x_{i}$ и $x_{j}$. Если связь последовательная (рис. 1), то

$$
\begin{gathered}
M_{i, j}=P_{n} \times P_{n-1} \times \ldots \times P_{2} \times P_{1} \\
\left(P_{1}, \ldots, P_{n}-\text { МП блоков } B_{1}, \ldots, B_{n}\right),
\end{gathered}
$$

Рис. 1.

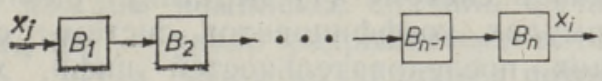

если параллельная (рис. 2), то

$$
M_{i, j}=P_{n} \times\left(P_{2}+P_{3}+\ldots+P_{n-1}\right) \times P_{1} \text {, }
$$

если более сложная, то матрицу $M_{i, j}$ можно найти комбинированием формул (2) и (3). Таким образом получаются все формулы коэффициентов системы (1) и сохраняются в памяти ЭВМ в виде последовательности цифр.

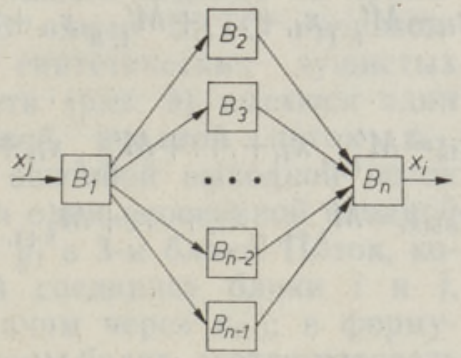

Рис. 2.

Однако полученная система еще не поддается решению, так как она содержит члены неосновных потоков, которые, в свою очередь, являются формулами и могут содержать потоки из правой и левой частей рассматриваемой системы. Следовательно, их надо из системы исключить, сложив их слагаемые с соответствующими членами системы уравнений. Для этого просматриваются формулы всех входных потоков начиная с $y_{1}$. Если в формуле входного потока $y_{l}(l=1, \ldots, t)$ поток $x_{j}$ входной или разрываемый, то во всех уравнениях системы находят новые формулы для коэффициентов потока $x_{j}$ :

$$
M_{k_{1}, j}^{\prime}=M_{k_{1}, j}+M_{k_{1}, k_{2}} \times P_{x /}
$$

где $M_{k_{1}, j}-$ прежний коэффициент потока $x_{j}$ в $k_{1}$-м уравнении $\left(k_{1}=1, \ldots, k+m+q\right), \quad M_{k_{1}, j}^{\prime}-$ новый коэффициент, $M_{k_{1}, k_{2}}-$ коэффициент потока $y_{l}$ в $k_{1}$-м уравнении $\left(k_{2}=1, \ldots, k+p+t\right), P_{x_{l}}-$ коэффициент потока $x_{j}$ в формуле входного потока $y_{l}$.

Если в формуле входного потока $y_{l}$ поток $x_{j}$ не является основным входным или разрываемым, то для него в системе (1) должно быть предусмотрено свое уравнение (в числе последних $q$ уравнений). Если некоторый коэффициент неосновных входных потоков в этом уравнении отличается от нуля, то поток $y_{l}$ в данный момент не учитывается. В противном случае поток $x_{j}$ в формуле потока заменяется этим уравнением. Если формула раньше имела вид

$$
y_{l}=P_{l_{1}} x_{n_{1}}+\ldots+P_{l_{l}} x_{j}+\ldots+P_{l_{v}} x_{n_{v}},
$$

где $x_{n_{1}}, \ldots, x_{n_{v}}$ - потоки комплекса, $P_{l_{1}}, \ldots, P_{l_{v}}-$ соответствующие им матрицы, то теперь она принимает вид

$$
y_{l}=P_{l_{1}} x_{n_{1}}+\ldots+P_{l J} M_{k+m+q_{1}, 1} x_{i_{1}}+\ldots+P_{l} M_{k+m+q_{1}, k+p} x_{\mathrm{Bx}_{p}}+\ldots+P_{l_{v}} x_{n_{v}}
$$


где $k+m+q_{1}-$ порядковый номер уравнения для $x_{j}$ в системе (1). Так поступают со всеми потоками в формуле для $y_{l}$, которые не являются основными входными или разрываемыми. После этого можно все члены потока $y_{l}$ сложить с первыми $k+p$ членами во всех уравнениях и считать коэффициенты формулы для $y_{l}$ во всех уравнениях равными нулю.

Во вторую очередь просматриваются неосновные входные потоки, которые в первый раз не учитывались. Некоторые потоки, возможно, придется пропустить и на этот раз. Тогда их надо просмотреть в третью очередь. и так до тех пор, пока коэффициенты всех неосновных входных потоков в системе (1) не станут равными нулю. Всем изменениям в формулах коэффициентов системы сопутствуют изменения соответствующих последовательностей цифр, хранящихся в памяти ЭВМ.

Получается следующая система линейных уравнений:

$$
\begin{aligned}
& x_{i_{1}}=M_{1,1}^{\prime} x_{i_{1}}+\ldots+M_{1, k}^{\prime} x_{i_{k}}+M_{1, k+1}^{\prime} x_{\mathrm{Bx}_{1}}+\ldots+M_{1, k+p}^{\prime} x_{\mathrm{Bx}_{p}}, \\
& x_{i_{k}}=M_{k, 1}^{\prime} x_{i_{1}}+\ldots+M_{k, k}^{\prime} x_{i_{k}}+M_{k, k+1}^{\prime} x_{\mathrm{Bx}_{1}}+\ldots+M_{k, k+p}^{\prime} x_{\mathrm{Bx}_{p}} \text {, } \\
& x_{\mathrm{Bdx}}=M_{k+1,1}^{\prime} x_{i_{1}}+\ldots+M_{k+1, k}^{\prime} x_{i_{k}}+M_{k+1, k+1}^{\prime} x_{\mathrm{Bx}_{4}}+\ldots+M_{k+1, k+p}^{\prime} x_{\mathrm{Bx}_{p}} \text {, }
\end{aligned}
$$

$$
\begin{aligned}
& x_{\mathrm{Bdx} m}=M_{k+m, 1}^{\prime} x_{i_{1}}+\ldots+M_{k+m, k}^{\prime} x_{i_{k}}+M_{k+m, k+1}^{\prime} x_{\mathrm{Bx}_{1}}+\ldots+M_{k+m, k+p}^{\prime} x_{\mathbf{B x}_{p}} \text {, } \\
& x_{j_{1}}=M_{k+m+1,1}^{\prime} x_{i_{1}}+\ldots+M_{k+m+1, k}^{\prime} x_{i_{k}}+M_{k+m+1, k+1}^{\prime} x_{\mathrm{BX}_{1}}+\ldots+ \\
& +M_{k+m+1, k+p}^{\prime} x_{\mathrm{Bx}_{p}} \\
& x_{j q}=M_{k+m+q, 1}^{\prime} x_{i_{1}}+\ldots+M_{k+m+q, k}^{\prime} x_{i_{k}}+M_{k+m+q, k+1}^{\prime} x_{\mathrm{Bx}_{1}}+\ldots+ \\
& +M_{k+m+q, k+p}^{\prime} x_{\mathrm{Bx}_{p}} \text {. }
\end{aligned}
$$

Для решения системы (4) нужно сперва решить систему линейных уравнений только из первых $k$ уравнений. Члены с основными входными потоками в этих уравнениях являются свободными членами. Система решается методом подстановки. Поток $x_{i_{k}}$ начиная с $k$-го уравнения выражается следующим образом:

$$
\begin{aligned}
x_{i_{k}}= & \left(E-M_{k, k}^{\prime}\right)^{-1}\left(M_{k, 1}^{\prime} x_{i_{1}}+\ldots+M_{k, k-1}^{\prime} x_{i_{k-1}}+M_{k, k+1}^{\prime} x_{\mathrm{Bx}_{1}}+\ldots+\right. \\
& \left.+M_{h, k+p}^{\prime} x_{\mathrm{Bx} p}\right)=\left(E-M_{k, k}^{\prime}\right)^{-1} M_{k, 1}^{\prime} x_{i_{1}}+\ldots+\left(E-M_{k, k}^{\prime}\right)^{-1} M_{k, k-1}^{\prime} x_{i_{k-1}}+ \\
& +\left(E-M_{k, k}^{\prime}\right)^{-1}\left(M_{k, k+1}^{\prime} x_{\mathrm{BX}_{1}}+\ldots+M_{k, k+p}^{\prime} x_{\mathrm{Bx}_{p}}\right),
\end{aligned}
$$

где $E$ - единичная матрица. В остальных $k-1$ уравнениях $x_{i_{k}}$ заменяется вышеприведенными уравнениями и изменяются все коэффициенты. Теперь получается система из $k-1$ уравнений с коэффициентами

$$
\begin{gathered}
M_{1,1}^{\prime \prime}, \ldots, M_{1, k-1}^{\prime \prime}, M_{1, k+1}^{\prime \prime}, \ldots, M_{1, k+p}^{\prime \prime}, \ldots, M_{k+m+q, 1}^{\prime \prime}, \\
\ldots, M_{k+m+q, k-1}^{\prime \prime}, M_{k+m+q, k+1}^{\prime \prime}, \ldots, M_{k+m+q, k+p}^{\prime \prime} .
\end{gathered}
$$


Из этой системы выражается $x_{i_{k-1}}$ и т.д. Последним остается уравнение

откуда

$$
x_{i_{1}}=M_{1,1}^{(k)} x_{i_{1}}+M_{1, k+1}^{(k)} x_{\mathrm{Bx}_{1}}+\ldots+M_{1, k+p}^{(k)} x_{\mathrm{Bx}_{p}}
$$

$$
x_{i_{1}}=\left(E-M_{1,1}^{(k)}\right)^{-1}\left(M_{1, k+1}^{(k)} x_{\mathrm{Bx}_{1}}+\ldots+M_{1, k+p}^{(k)} x_{\mathrm{Bx}_{p}}\right) .
$$

После этого остальные $q+m$ уравнений системы (4) являются формулами уже известных потоков. Таким образом получаются формулы, соединяющие входные и выходные потоки комплекса, т. е. ЭМП одного комплекса.

На втором шаге расчет по разработанным формулам производится тоже по комплексам. В каждом комплексе сначала вычисляются матрицы в формуле для $x_{i_{k}}(k-$ число разрываемых потоков в комплексе), в формуле для $x_{i_{k-1}}$ и т. д. Затем вычисляются потоки в порядке $x_{i}, \ldots, x_{i_{k}}, x_{\text {вых }}, \ldots, x_{\text {вых }_{m}}, x_{j_{1}}, \ldots, x_{j q}$.
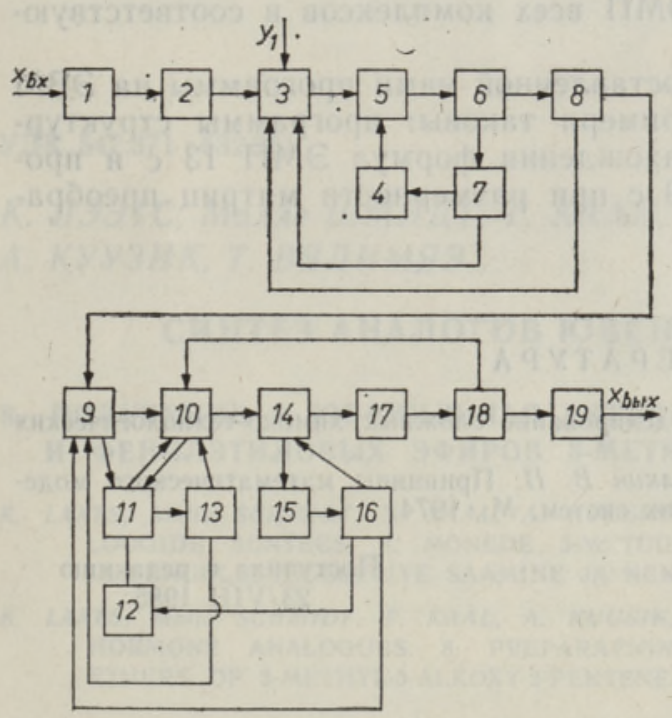

Рис. 3,

Рассмотрим принципы и технику нашего подхода на примере. В схеме СХТС производства синтетических душистых веществ (рис. 3) имеются один основной входной поток $x_{\mathrm{Bx}}$, один основной выходной поток $x_{\text {вых }}$ и один неосновной входной поток $y_{1}$ в 3-м блоке. Поток, который соединяет блоки $i$ и $j$, обозначим через $x_{i, j}$; в формулах ему будет соответствовать цифра - $(100 \cdot i+j)$. ЭМП этого потока обозначим через $P_{i, j}$; в формулах ей будет соответствовать цифра $100 \cdot i+j$. Символы матричных операций: 100 умножение, 200 - сложение, 300 - обращение матриц, 400 вычитание.

Из сущности рассматриваемого процесса вытекает, что

$$
y_{1}=R_{11} \times\left(x_{2,3}+x_{7,3}+x_{8,3}\right),
$$

где $R_{11}$ - определенная матрица. Формула потока $y_{1}$ вводится в ЭВМ и сохраняется в виде следующей последовательности цифр: 51, - 203, $100,51,-703,100,200,51,-803,100,200$, где 51 - символ матрицы $R_{11}$.

Структурным анализом находится, что схема состоит изุ пяти комплексов: 1 - блок $1 ; 2$ - блок $2 ; 3$ - блоки $3-8 ; 4-$ блоки $9-18$ и 5 - блок 19. В схеме пять разрываемых потоков: $x_{5,6}, x_{10,11}, x_{10,14}$, $x_{11,13}$ и $x_{14,15}$.

Рассмотрим подробнее третий комплекс. Программа строит первоначальную систему линейных уравнений и преобразует еe, так как в правых частях находятся члены с $y_{1}$. После преобразования получается:

$$
\begin{aligned}
& x_{5,6}=M_{1,1}^{\prime} x_{5,6}+M_{1,2}^{\prime} x_{2,3}, \\
& x_{8,9}=M_{2,1}^{\prime} x_{5,6}+M_{2,2}^{\prime} x_{2,3}, \\
& x_{7,3}=M_{3,1}^{\prime} x_{5,6}+M_{3,2}^{\prime} x_{2,3}, \\
& x_{8,3}=M_{4,1}^{\prime} x_{5,6}+M_{4,2}^{\prime} x_{2,3} .
\end{aligned}
$$


Все формулы коэффициентов $M_{i, j}^{\prime}(i=1,2,3,4 ; j=1,2)$ сохра няются в памяти ЭВМ. Например, $M_{1,2}^{\prime}$ соответствует последовательность цифр: $506,305,100,506,305,100,51,100,200$. Здесь система линейных уравнений состоит только из одного (первого) уравнения $\left(x_{2,3}-\right.$ основной входной поток в комплекс). Получается: $x_{5,6}=$ $=\left(E-M_{1,1}^{\prime}\right)^{-1} \cdot M_{1,2}^{\prime} x_{2,3}$, которая сохраняется в памяти ЭВМ в виде последовательности: $11111,10101,400,300,10102,-203,100,100$, где 11111 - символ единичной матрицы $E, 10101$ и 10102 - символы $M_{1,1}^{\prime}$ и $M_{1,2}^{\prime}$.

При расчете ЭМП данного комплекса по найденным формулам вычисляются сначала коэффициенты в порядке $M_{1,1}^{\prime} ; M_{1,2}^{\prime}, M_{2,1}^{\prime}, M_{2,2}^{\prime}$, $M_{3,1}^{\prime}, M_{3,2}^{\prime} ; M_{4,1}^{\prime}, M_{4,2}^{\prime} \quad$ (эти формулы зависят только от $M П$ отдельных блоков), после чего рассчитываются $x_{5,6}, x_{8,9}, x_{7,3}$ и $x_{8,3}$. Расчет потока $x_{8,9}$ дает ЭМП данного комплекса. ЭМП целой системы получается умножением найденных ЭМП всех комплексов в соответствующем порядке.

Скоростные характеристики составленной нами программы на ЭВМ EC-1022 для вышеописанного примера таковы: программы структурного анализа 2 с, программы нахождения формул ЭМП 13 с и программы расчета по формулам 59 с при размерности матриц преобразования $10 \times 10$.

\section{ЛИТ Е Р А Т Р А}

1. Островский Г. М., Волин Ю. М. Моделирование сложных химико-технологических схем. М., 1975.

2. Кафаров В. В., Перов В. Л., Мешалкин В. П. Принципы математического моделирования химико-технологических систем. М., 1974.
Ннститут химии
Академии наук Эстонской ССР
Поступила в редакцию 23/VIII 1985

\section{R. KASK, I. KIRJANEN}

\section{KEERULISTE KEEMILIS-TEHNOLOOGILISTE SUSTEEMIDE ARVUTAMISE MAATRIKSMEETOD}

Artiklis on kirjeldatud maatriksmeetodit keeruliste keemilis-tehnoloogiliste süsteemide ekvivalentsmaatriksi arvutamiseks. Meetod on iteratsioonivaba, hästi programmeeritav ja võimaldab ekvivalentsmaatriksi arvutamise täielikult formaliseerida. Arvutamine toimub kahes etapis. Alguses leitakse arvutusvalemid, mis sõltuvad ainult süsteemi struktuurist, pärast arvutatakse nende valemite pōhjal. Seesugune moodus on eriti ökonoomne, kui süsteemi on tarvis arvutada mitu korda (näiteks optimeerimise korral). Artikli lōpul on toodud näide mieetodi kasutamisest.

\section{R. KASK, I. KIRJANEN}

\section{A METHOD FOR COMPUTING COMPLICATED CHEMICAL-TECHNOLOGICAL SYSTEMS}

A matrix-method for computing the equivalent-matrix of complicated chemical-technological systems is presented. The method is iteration-free and can be relatively easily programmed, making it possible to formalize completely the computation of the equivalent-matrix. Computation proceeds in two stages: firstly, the formulas of calculation are found (they depend only on the structure of the system), and secondly, the system is calculated by these formulas. Such a mode is particularly economical for calculating the system many times over (for example, in case of optimization). An example of using the method is presented at the end of the paper. 